\title{
Impact of Preclinical Labor and Delivery Shadowing on Student Perceptions of Obstetrics and Gynecology as a Specialty and Possible Career: A Prospective Cohort
}

\author{
Sarah K. Dotters-Katz, MD, MMHPE ${ }^{1} \quad$ Marcela C. Smid, MD² Sara Tinkham³ Alice Chuang, MD, MEd ${ }^{3}$ \\ ${ }^{1}$ Department of Obstetrics and Gynecology, Duke University, \\ Durham, North Carolina \\ 2 Department of Obstetrics and Gynecology, University of Utah, Salt \\ Lake City, Utah \\ ${ }^{3}$ Department of Obstetrics and Gynecology, University of North \\ Carolina, Chapel Hill, North Carolina

\begin{abstract}
Address for correspondence Sarah K. Dotters-Katz, MD, Department of Obstetrics and Gynecology, Duke Maternal Fetal Medicine, 2608
\end{abstract} \\ Erwin Road, STE 210, Durham, NC 27710 (e-mail: sd132@duke.edu).
}

Am J Perinatol Rep 2019;9:e361-e365.

\begin{abstract}
Keywords

- labor and delivery

- shadowing

- preclinical exposure

- medical students

- perceptions

- obstetrics

- culture

Objective The purpose of this study was to determine the impact of preclinical shadowing on student interest and perceptions of obstetrics and gynecology (OBGYN). Methods We enrolled a prospective cohort of preclinical medical students who shadowed on labor and delivery (L\&D). Students sent electronic surveys a week prior (presurvey), the week after (postsurvey), and three months after shadowing (farsurvey). Responses compared using descriptive statistics. We analyzed common themes of free text responses.

Results From July 2016 to April 2017, 41 students shadowed on L\&D; 81\% were female. Eighty percent responded to at least one survey, $37 \%$ completed all surveys, (presurvey: $76 \%$, postsurvey: $51 \%$, and far-survey: $46 \%$ ). Prior to shadowing, $10 \%$ (3/31) planed a career in OBGYN compared with $24 \%(5 / 21)$ after shadowing $(p=0.42)$. Over $50 \%$ of students described the people and procedures as altering their perceptions of OBGYN in a positive way. Common themes explaining this change included: culture $(n=4)$, team interactions $(n=4)$, seeing deliveries $(n=3)$, and hands-on experiences $(n=3)$. Three months after shadowing, $79 \%$ described the experience as very worthwhile. Eighty-nine percent would recommend the experience to a friend not interested in OBGYN and $100 \%$ stated they would shadow again.

Discussion Although shadowing may not increase students' desire to pursue OBGYN, it is nearly universally felt to be worthwhile and improves perceptions of the field.
\end{abstract}

Many medical schools are moving to increase clinical opportunities in the preclinical years. However, even with these changes, shadowing in the preclinical years provides contact with fields and clinical arenas that most students have never seen before. Preclinical medical students, through shadow- ing, thus gain insight into various careers, even before starting their clinical clerkships.

These experiences have the power to shape, both negatively and positively a student's perceptions about a field and, more importantly, their future careers. In emergency received

December 27, 2018 accepted after revision May 25, 2019
DOI https://doi.org/

10.1055/s-0039-1695747. ISSN 2157-6998.
Copyright $\odot 2019$ by Thieme Medical Publishers, Inc., 333 Seventh Avenue, New York, NY 10001, USA. Tel: +1(212) 584-4662.
License terms

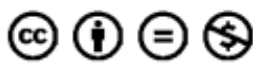


medicine (EM), at one center that surveyed shadowing students in the emergency department, $29 \%$ were less interested in a career in EM after the experience and $24 \%$ were more interested. ${ }^{1}$ In another similar study, also in EM, $67 \%$ of students commented that their attitudes and interest about the field were changed by the shadowing experiences. ${ }^{2}$ Shadowing may be especially beneficial for fields perceived by students to have a "challenging" lifestyle, such as surgery or obstetrics and gynecology (OBGYN).

In the surgery literature, there is ample data suggest that students shadowing provide very useful exposure and insight into the specialty and may even increase rates of students choosing surgery as a career choice. ${ }^{3-6}$ However, similar data from OBGYN is lacking. Though one recently published study of shadowing experiences on labor and delivery (L\&D) suggested that this may increase student interest in OBGYN but was limited in that the survey was administered up to 2 years after the experience in some cases. ${ }^{7}$ Thus, the purpose of this study was to prospectively assess the impact of shadowing on L\&D on students attitudes about OBGYN as a specialty and interest in this as a career choice.

\section{Methods}

After obtaining exemption from the Institutional Review Board at the University of North Carolina at Chapel Hill Office of Human Research Ethics, we conducted a three-part prospective survey of first year medical students at the University of North Carolina who took part in an optional shadow experience on the L\&D unit between July 2016 and June 2017.

We designed three surveys, one for students to take within a week prior to shadowing, one to take within a week after shadowing, and one to take 3 months after shadowing. The surveys were edited and refined by a qualitative methods expert (Teresa Edwards) at the Howard W. Odum Institute for Research in Social Science at the University of North Carolina. Each survey was then piloted with a small group of students for clarity and conciseness. The first survey (presurvey) had five demographic questions, two questions about future career plan, and three questions specifically about shadowing, and two questions about their perceptions of OBGYN. The second survey (postsurvey) had three questions specifically about what the student saw/did during the time on $L \& D$, four questions about their perceptions of OBGYN, and four questions about the value of the experience. The final survey (far-survey) asked three questions about career plans, three about perceptions of OBGYN, and four about the value of the experience. At all three time points, students were asked on a scale of 1 to 5 , what their likelihood of choosing a career in OBGYN was, with 1 corresponding to "I'm certain that I will be an OBGYN" and 5 to "I am sure that OBGYN is NOT for me."

Each student who signed up through the OBGYN student interest group to shadow received an e-mail with instructions from the coordinator a few days prior to their scheduled shadow date. That e-mail was also a brief explanation of our survey and a "heads up" about the e-mails they would be receiving. The surveys were entered into Qualtrics (Qualtrics, Provo, UT), an electronic survey tool. Each student received an e-mail with a link to the presurvey within a week prior to shadowing, a second e-mail with a link to the postsurvey within a week after shadowing, and finally a third e-mail with a link to the far-survey 3 months after shadowing. For the postsurvey and far-survey, a reminder e-mail was sent 1 week after the original e-mail if the student had not completed the survey.

Only students who completed at least one survey were included in the analysis. To link each student's pre-, post-, and far-survey, a third party compiled the data using e-mail addresses and then deidentified it, prior to any analysis. Within the survey, students were asked to describe their time on L\&D as "busy" or "slow." Students who shadowed on self-described "busy" days were compared with those who shadowed on self-described "slow" days. Chi-square, $t$-tests, Wilcoxon's rank sum test, and Fischer's exact test were used as appropriate to describe data within each survey. Paired $t$ tests were used to compare students' responses between surveys. If a student only completed one survey, their data were not included in paired comparisons. STATA, version 13 (College Station, TX), was used to analyzed the data. Qualitative analysis was used to identify common themes those were analyzed from free text responses.

\section{Results}

Forty-one students shadowed on L\&D during the study period, of those, 31 (76\%) completed the presurvey, 21 (51\%) the postsurvey, and 19 (46\%) the far-survey. A total of $33(80 \%)$ students completed at least one survey, 23 (56\%) completed at least two surveys, and 15 (37\%) did all three surveys. Overall, the respondent population was $82 \%$ female, $29 \%$ had shadowed before and over half of them were considering OBGYN as a career prior to shadowing (-Table 1). Among the 21 students who completed the postsurvey, 13 described the day as "busy." Students who shadowed on slow days did not differ demographically from those who shadowed on "busy days" with regards to gender, age, prior shadowing experience, or interest in OBGYN as a career (-Table 1). Vaginal deliveries, rounding, and seeing patient in triage did not differ in frequency between these two groups (-Table 1). However, students who shadowed on busy day were more likely to see a delivery of some kind (100 vs. $50 \%, p=0.01$ ) and to see a cesarean section (77 vs. $13 \%$, $p<0.01$ ).

- Table 2 describes students' responses to questions about the impact of shadowing on their perceptions of OBGYN and likelihood of choosing OBGYN as a career. Students who shadowed on a slow day did not feel that their time on L\&D effected their desire to pursue a career in OBGYN ( $p=$ 0.05 ). In contrast, student perceptions of OBGYN were more likely to be affected in a positive way by people and procedures on busy days compared with slow days, $(p=0.03$ and $p<0.01$, respectively, - Table 2). Among all students who shadowed, $86 \%$ would recommend the experience to a friend 
Table 1 Demographic information and shadowing experiences from students completing at least one survey regarding the L\&D shadow experience, and comparing respondents who shadowed on busy versus slow days

\begin{tabular}{|c|c|c|c|c|}
\hline & $\begin{array}{l}\text { Entire population of } \\
\text { respondents } \\
n=33(\%)\end{array}$ & $\begin{array}{l}\text { Busy }^{c} \\
n=13 \text { (\%) }\end{array}$ & $\begin{array}{l}\text { Not busy } \\
n=8(\%)\end{array}$ & $p$-Value \\
\hline Female gender & $27(82)$ & $11(85)$ & $4(50)$ & 0.15 \\
\hline Mean age, $y,( \pm S D)$ & $24( \pm 2.7)$ & $24( \pm 2.0)$ & $25( \pm 4.6)$ & 0.60 \\
\hline Shadowed before & $9(29)$ & $9(69)$ & $4(50)$ & 0.65 \\
\hline Completed all three surveys & $15(45)$ & $10(77)$ & $5(63)$ & 0.63 \\
\hline Considering OBGYN as a career prior to shadowing & $17(52)$ & $7(54)$ & $4(50)$ & $>0.99$ \\
\hline \multicolumn{5}{|l|}{ Shadowing experiences $^{\mathrm{a}}$} \\
\hline Saw any delivery & $17(81)$ & $13(100)$ & $4(50)$ & 0.01 \\
\hline Saw vaginal delivery & $9(43)$ & $6(46)$ & $3(38)$ & $>0.99$ \\
\hline Saw Cesarean delivery & $11(52)$ & $10(77)$ & $1(13)$ & 0.008 \\
\hline Rounded with team & $10(48)$ & $6(46)$ & $4(50)$ & $>0.99$ \\
\hline Saw a triage patient & $6(29)$ & $4(31)$ & $2(25)$ & $>0.99$ \\
\hline Saw two activities ${ }^{\mathrm{b}}$ & $9(43)$ & $7(54)$ & $2(22)$ & 0.37 \\
\hline Spent majority of time with attending & $10(47)$ & $7(54)$ & $3(38)$ & 0.66 \\
\hline
\end{tabular}

Abbreviations: L\&D, labor and delivery; OBGYN, obstetrics and gynecology; SD, standard deviation.

${ }^{\mathrm{a}}$ Only includes respondents of postsurvey $(n=21)$.

${ }^{\mathrm{b}}$ Activities defined as: vaginal delivery, Cesarean delivery, rounds, or triage patient.

'Only 21 students who completed the post survey were considered for busy/not-busy comparison. There were no differences in those who did and did not complete the posttest.

who was not interested in OBGYN. Additionally, for all participants, likelihood of becoming an OBGYN increased after shadowing (mean change $=-0.39$, standard deviation $[S D]=0.70$ ) When compared with those who shadowed in a slow day, shadowing on a busy day was more impactful with regards to a student's likelihood of pursuing a career in OBGYN (mean change $=-0.67, \mathrm{SD}=0.65$ vs. $0.17, \mathrm{SD}=$ $0.41, p=0.01)$.

In contrast, by 3 months out, this effect had declined. Among all students who completed the far-survey, there was no change in a likelihood of pursuing a career in OBGYN from prior to shadowing (average change $=0, S D=0.61$ ). When comparing students who shadowed on busy days to those on slow days, the difference has dissipated as well (-Table $\mathbf{3}$ ). However, students were still overwhelmingly described the experience as worthwhile (95\%) with 79\% describing it as very worthwhile. Nearly $90 \%$ of students said they would recommend to a friend not interested in OBGYN, including $83 \%$ of students who shadowed on slow days. All students stated they would shadow again if they had the chance.

Within the qualitative analysis of the postsurvey, when asked what factors made a difference in their likelihood to

Table 2 Student responses from postsurvey regarding the impact of shadowing on their perceptions of OBGYN

\begin{tabular}{|l|l|l|l|l|}
\hline & $\begin{array}{l}\text { Entire population } \\
\text { of respondents } \\
n=21(\%)\end{array}$ & $\begin{array}{l}\text { Busy } \\
\boldsymbol{n}=13(\%)\end{array}$ & Not busy $\boldsymbol{n = 8}(\%)$ & $p$-Value \\
\hline $\begin{array}{l}\text { After spending time on L\&D, I am more likely } \\
\text { pursue a career in OBGYN }\end{array}$ & $15(71)$ & $11(85)$ & $4(50)$ & 0.15 \\
\hline $\begin{array}{l}\text { Spending time on L\&D, did not make difference } \\
\text { in how likely I am to pursue a career in OBGYN }\end{array}$ & $5(24)$ & $1(8)$ & $4(50)$ & 0.05 \\
\hline $\begin{array}{l}\text { People I worked with altered my opinion in very } \\
\text { positive way }\end{array}$ & $12(57)$ & $10(77)$ & $2(25)$ & 0.03 \\
\hline $\begin{array}{l}\text { Procedures I saw altered opinion in very posi- } \\
\text { tive way }\end{array}$ & $11(52)$ & $9(73)$ & $4(50)$ & $<0.01$ \\
\hline $\begin{array}{l}\text { Considering a career in OBGYN after } \\
\text { shadowing }\end{array}$ & $13(62)$ & $-0.67(0.65)$ & $0.17(0.41)$ & 0.65 \\
\hline $\begin{array}{l}\text { Average change in likelihood of pursuing } \\
\text { OBGYNa(SD) }\end{array}$ & $-0.39(0.70)$ & 0.01 \\
\hline
\end{tabular}

Abbreviations: L\&D, labor and delivery; OBGYN, obstetrics and gynecology; SD, standard deviation.

${ }^{a}$ Responses on a Likert's scale, $1=\mathrm{I}$ am certain that I will be an OBGYN and $5=\mathrm{I}$ am sure that OBGYN is not for me. 
Table 3 Student responses from far-survey regarding the impact of shadowing on their perceptions of OBGYN

\begin{tabular}{|l|l|l|l|l|}
\hline & $\begin{array}{l}\text { Entire population of } \\
\text { respondents } \\
\boldsymbol{n}=\mathbf{1 9}(\%)\end{array}$ & $\begin{array}{l}\text { Busy } \\
\boldsymbol{n}=\mathbf{1 0}(\%)\end{array}$ & $\begin{array}{l}\text { Not busy } \\
\boldsymbol{n}=\mathbf{6}(\%)\end{array}$ & $p$-Value \\
\hline Considering a career in OBGYN & $7(44)$ & $6(60)$ & $1(17)$ & 0.15 \\
\hline $\begin{array}{l}\text { Shadowing very much enhanced my } \\
\text { understanding of L\&D }\end{array}$ & $11(69)$ & $8(80)$ & $3(50)$ & 0.30 \\
\hline The experience was very worthwhile & $12(75)$ & $9(90)$ & $3(50)$ & 0.12 \\
\hline $\begin{array}{l}\text { I would recommend to a friend not interested } \\
\text { in OBGYN }\end{array}$ & $15(94)$ & $10(100)$ & $5(83)$ & 0.38 \\
\hline $\begin{array}{l}\text { Average change in likelihood of pursuing OB- } \\
\text { GYN since immediately after shadowing }\end{array}$ & $0.5(0.76)$ & $0.67(0.71)$ & $0.2(0.84)$ & 0.29 \\
\hline $\begin{array}{l}\text { Average change in likelihood of pursuing OB- } \\
\text { GYN prior to shadowing }\end{array}$ & $0.07(0.59)$ & $0(0.47)$ & $0.2(0.84)$ & 0.56 \\
\hline
\end{tabular}

Abbreviations: L\&D, labor and delivery; OBGYN, obstetrics and gynecology; SD, standard deviation.

${ }^{a}$ There were nine students who responded to both post- and far-survey in the busy group, and five in the slow group.

'There were 10 student who responded to both pre- and far-surveys in the busy group, and five in the slow group.

'Responses on a Likert's scale, $1=\mathrm{I}$ am certain that I will be an OBGYN and $5=\mathrm{I}$ am sure that OBGYN is not for me.

pursue a career in OBGYN, the common themes that emerged included: exposure to the field $(n=6)$, "good culture" $(n=4)$, and positive interactions with the team $(n=4)$. There were four students who reported being more likely to report a career in OBGNY after shadowing, but did not respond as to why. Themes did not differ between students who shadowed on busy compared with slow days. Representative examples of these themes can be seen in - Table 4. In the far-survey, when asked about the most meaningful aspect of their experience, 10 students mentioned exposure to the field, and five mentioned interactions with the team ( - Table 4$)$.

\section{Discussion}

In this prospective study, students shadow experiences on L\&D did not impact medical students' likelihood of going into
OBGYN. However, students noted that both the people they met and the procedures they saw while shadowing, changed their perceptions in an overwhelmingly positive way. Additionally, the vast majority found the experience very worthwhile and nearly all would recommend to a friend who was not interested in OBGYN.

Throughout the preclinical and clinical years of medical school, students have exposures and experiences almost daily that have the power to impact their perceptions of a field and ultimately career choice. Because OBGYN is often associated with some negative stereotypes (malpractice, long hours, etc.), any opportunity to help change that image early in medical school is be valuable. ${ }^{8}$ In 2005, American Congress of Obstetrics and Gynecology and Association of Professors of Gynecology and Obstetrics emphasized the importance of early exposure through interest groups and

Table 4 Themes and representative quotes

\begin{tabular}{|c|c|}
\hline Postsurvey & \\
\hline $\begin{array}{l}\text { What made you more likely to pursue a career in OBGYN } \\
\text { after shadowing? }^{\text {a }}\end{array}$ & Examples \\
\hline Exposure to the field $(n=6)$ & $\begin{array}{l}\text { "Being hands-on experience in the delivery process helped me } \\
\text { realize that I would love to have it be a part of my career" }\end{array}$ \\
\hline Good culture $(n=5)$ & $\begin{array}{l}\text { "The demeanor of the interns, the chief resident, and the } \\
\text { attending. They seemed so happy and really enjoyed } \\
\text { their job" }\end{array}$ \\
\hline Interactions with the team $(n=4)$ & $\begin{array}{l}\text { "Getting to talk to the attendings and residents and learn about } \\
\text { their experiences" }\end{array}$ \\
\hline \multicolumn{2}{|l|}{ Far-survey } \\
\hline $\begin{array}{l}\text { What was the most meaningful aspect of your } \\
\text { experience? }\end{array}$ & Examples \\
\hline Exposure to the field $(n=10)$ & $\begin{array}{l}\text { "I got experience that I had not had previously" } \\
\text { "Seeing the live birth was awesome" }\end{array}$ \\
\hline Interactions with the team $(n=5)$ & $\begin{array}{l}\text { "Getting to talk to the attendings and residents and learn about } \\
\text { their experiences" }\end{array}$ \\
\hline
\end{tabular}


early career mentoring. ${ }^{8}$ Our data suggest that interactions with people during shadowing positively impacted perceptions of OBGYN (both quantitatively and qualitatively), hopefully helping to dispel those negative myths.

The data regarding interest groups and impact on career choice is mixed. Data from family medicine interest groups suggest that in positively impacts perceptions of the field but does not actually increase family medicine as a career choice. ${ }^{9,10}$ However, Blau et al found that participation in a rural medicine interest group was impactful on career choice. ${ }^{11}$ While this is not specialty specific, it does suggest some impact on interest group participation. We were unable to identify any data regarding OBGYN interest group participation and ultimate career choice. ${ }^{11}$ However, Hammoud et al surveyed nearly 270 third year medical students after their OBGYN clerkship, and found that the best predicator of interest postclerkship was interest preclerkship. ${ }^{12}$ This further underscores the importance of preclinical exposure to OBGYN.

Though shadowing on L\&D is nearly ubiquitous at academic medical centers, data regarding its value are quite limited. This study adds to the growing, albeit petite, body of literature on this subject. Additionally, another strength of this study is the prospective nature of the study, as well as assessing student perceptions not only before and after, but at a third time point, 3 months after the initial experience. However, our study is not without limitations. The biggest limitation of this study is the low number of students. Our clerkship changed format at the same time that we began our study, requiring students to work on Saturday mornings. Thus, the number of student spots for shadowing was halved. In other words, each weekend, there was only one student shadow spot instead of the two we had had the prior year. We are also limited by our response rate. This low number also limited the statistical analysis which we could perform. Thus, this study is really hypothesis generating and we are unable to assess causation. No validated instrument existed for this sort of survey either. However, by obtaining help in creating our instruments from an expert in survey design and qualitative methods, we hoped to mitigate this somewhat. Surveys are also always at risk for respondent bias as well.

\section{Conclusion}

A myriad of preclinical experiences exist in medical education. Data from interest groups suggest that this sort of experience may not influence career choice but provides a

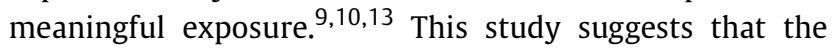
opportunity to shadow on L\&D is a similarly meaningful experience for students. More importantly, our data suggest that this preclinical exposure improves perceptions of OBGYN, hopefully decreasing stereotypes of our specialty and anxiety surrounding the clerkship. Further prospective and longitudinal studies are needed to better assess the impact of these experiences on matched specialty, as well as focus groups, with students to understand specifically what aspects of this experience make it valuable. However, preclinical exposure to L\&D is valuable experience for students, which should continue to be supported by OBGYN departments as it meaningfully impacts students' perceptions of the specialty.

\section{Prior Presentation}

This research was presented in part as a poster at the 2018 APGO CREOG meeting in National Harbor, MD.

\section{Funding \\ None.}

\section{Conflict of Interest}

The authors report no conflict of interest.

\section{Acknowledgments}

The authors would like to acknowledge the Teresa Edward at the Odum Institute at the University of North Carolina for the help in survey development.

\section{References}

1 Gharahbaghian L, Hindiyeh R, Langdorf MI, et al. The effect of emergency department observational experience on medical student interest in emergency medicine. J Emerg Med 2011;40 (04):458-462

2 Penciner R. Emergency medicine preclerkship observerships: evaluation of a structured experience. CJEM 2009;11(03): 235-239

3 Foote DC, Meza JM, Sood V, Reddy RM. Assessment of female medical students' interest in careers in cardiothoracic surgery. J Surg Educ 2017;74(05):811-819

4 Antiel RM, Thompson SM, Camp CL, Thompson GB, Farley DR. Attracting students to surgical careers: preclinical surgical experience. J Surg Educ 2012;69(03):301-305

5 Cloyd J, Holtzman D, O'Sullivan P, Sammann A, Tendick F, Ascher N. Operating room assist: surgical mentorship and operating room experience for preclerkship medical students. J Surg Educ 2008;65(04):275-282

6 Drolet BC, Sangisetty S, Mulvaney PM, Ryder BA, Cioffi WG. A mentorship-based preclinical elective increases exposure, confidence, and interest in surgery. Am J Surg 2014;207(02):179-186

7 Dotters-Katz S, Panzer A, Givens M, Smid M, Chuang A. Preclinical labor-and-delivery shadowing: the impact on medical students' perceptions of obstetrics and gynecology. AJP Rep 2018;8(02): e64-e67

8 Bienstock JL, Laube DW. The recruitment phoenix: strategies for attracting medical students into obstetrics and gynecology. Obstet Gynecol 2005;105(5, Pt. 1):1125-1127

9 Wei McIntosh E, Morley CP. Family medicine or primary care residency selection: effects of family medicine interest groups, $\mathrm{MD} / \mathrm{MPH}$ dual degrees, and rural medical education. Fam Med 2016;48(05):385-388

10 Hinchey S, LaRochelle J, Maurer D, Shimeall WT, Durning SJ, DeZee $\mathrm{KJ}$. Association between interest group participation and choice of residency. Fam Med 2011;43(09):648-652

11 Blau EM, Aird P, Dolovich L, Burns S, del Pilar-Chacon M. Rural medicine interest groups at McMaster University: a pilot study. Can J Rural Med 2009;14(04):139-144

12 Hammoud MM, Stansfield RB, Katz NT, Dugoff L, McCarthy J, White CB. The effect of the obstetrics and gynecology clerkship on students' interest in a career in obstetrics and gynecology. Am J Obstet Gynecol 2006;195(05):1422-1426

13 Quirk SK, Riemer C, Beers PJ, et al. Dermatology interest groups in medical schools. Dermatol Online J 2016;22(07):13030/ qt1zm883qs 